\title{
Effect of humidity on the wooden beams of the composite section
}

\author{
Vladimir Linkov ${ }^{1, *}$ \\ ${ }^{1}$ Moscow State University of Civil Engineering, 129337, 26, Yaroslavskoye Shosse, Moscow, Russia
}

\begin{abstract}
In wooden structures, the change in humidity affects the deformability and load-carrying capacity of joints and structures according to the criteria of the first and second groups of limiting states. In wooden beams of the composite cross-section with mechanical connections, with an increase in the moisture content of the timber, the structural strength of the structures is affected both by the increment in the deformations of the joints and by the change in the physical and mechanical characteristics of the wood, primarily, the modulus of elasticity of the material.
\end{abstract}

\section{Introduction}

It is very important to check the deformability of cross-section wooden elements [1-3]. The theory of compound rods by A.R. Rzhanitsyn is used in this article. Let's consider a beam with a span of $6 \mathrm{~m}$ with a cross-section $\mathrm{bxh}=150 \times 450 \mathrm{~mm}$ from three bars $150 \times 150$ $\mathrm{mm}$, the joint operation of which is provided by inclined metal rods of $\mathrm{d}=20 \mathrm{~mm}$ set at an angle of 45 degrees to the longitudinal axis of the beam and to the direction of the fibers in the bars of the composite section. Load on the beam is transmitted in the form of two concentrated forces, applied symmetrically in a $1 / 4$ span of the structure. Inclined rods are installed on three pieces from each side of the beam in areas where shear forces act in the joints between the bars of the composite section. The pitch of the rods is $450 \mathrm{~mm}$.

\section{Materials and Methods}

According to Rzhaynitsyn's theory of compound rods that is mirrored in later works [4-6], the beam design consisting of three separate rods connected by compliant shear links forms a statically indeterminate system, one of the main characteristics of which are deformations of compliant bonds obtained from experiments with constructions of full-scale sizes. Two differential equations (1) for seams of a three-layer beam

$$
\frac{T_{1}^{\prime \prime}}{\xi_{1}}=\Delta_{11} \times T_{1}+\Delta_{12} \times T_{2}+\Delta_{10}
$$

\footnotetext{
* Corresponding author: linkov-kdip@email.ru
} 


$$
\frac{T_{2}^{\prime \prime}}{\xi_{2}}=\Delta_{21} \times T_{1}+\Delta_{22} \times T_{2}+\Delta_{20}
$$

Coefficients of differential equations

$$
\begin{gathered}
\Delta_{11}=\Delta_{22}=\frac{2}{E I}+\frac{h^{2}}{3 E I}=\frac{\left(2+\frac{h^{2}}{3 i^{2}}\right)}{E F} \\
\Delta_{12}=\Delta_{21}=-\frac{1}{E F}+\frac{h^{2}}{3 E I}=\frac{\left(\frac{h^{2}}{3 i^{2}}-1\right)}{E F} \\
\Delta_{10}=\Delta_{20}=-\frac{M^{0}}{3 E I} \times h=-\frac{M^{0}}{3 i^{2}} \times h / E F
\end{gathered}
$$

where $\mathrm{Ti}$ - shearing force in the $\mathrm{i}$-th seam; $\mathrm{h}$ - the distance between the centers of gravity of the cross sections of two adjacent rods; M0 - bending moment in a beam that does not have shear bonds; $\mathrm{i}$ - radius of inertia of the section of each beam; $\xi \mathrm{i}$ - shear rigidity ratio in the i-th seam, $\xi \mathrm{i}=\mathrm{Tc} *{ }^{*} \mathrm{~m} / \delta \mathrm{c}$; Тст - shearing force per bond; $\delta \mathrm{c}$ - deformation of mutual displacement of two adjacent bars, obtained in the experiment; $\mathrm{m}$ - the number of links per unit length of the seam.

We substitute the coefficients (2) into the system (1):

$$
\begin{aligned}
& T_{1}^{\prime \prime} \frac{E F}{\xi_{1}}=\left(\frac{h^{2}}{3 i^{2}}+2\right) T_{1}+\left(\frac{h^{2}}{3 i^{2}}-1\right) \times T_{2}-\frac{M^{0} h}{3 i^{2}} \\
& T_{2}^{\prime \prime} \frac{E F}{\xi_{2}}=\left(\frac{h^{2}}{3 i^{2}}-1\right) T_{1}+\left(\frac{h^{2}}{3 i^{2}}+2\right) \times T_{2}-\frac{M^{0} h}{3 i^{2}}
\end{aligned}
$$

We introduce the notation $\beta=\frac{E F}{\xi}, \alpha=\frac{h^{2}}{3 i^{2}}, \quad \mu=-\frac{M^{0} h}{3 i^{2}}$.

Then the system (3) takes the form:

$$
\begin{aligned}
& \beta \times{T_{1}^{\prime \prime}}^{\prime \prime}(\alpha+2) T_{1}+(\alpha-1) \times T_{2}+\mu \\
& \beta \times{T_{2}^{\prime \prime}}^{\prime \prime}=(\alpha-1) T_{1}+(\alpha+2) \times T_{2}+\mu
\end{aligned}
$$

In our case, for three identical bars $\alpha=\frac{12 \times b h \times h^{2}}{3 \times b h^{3}}=4$.

Then

$$
\begin{aligned}
& \beta \times{T_{1}^{\prime \prime}}^{\prime \prime} 6 T_{1}+3 T_{2}+\mu \\
& \beta \times{T_{2}^{\prime \prime}}^{\prime \prime}=3 T_{1}+6 T_{2}+\mu
\end{aligned}
$$

Let us compose the sum and difference of these equations.

The system (4) takes the form

$$
\beta \times\left(T_{1}^{\prime \prime}+T_{2}^{\prime \prime}\right)=9\left(T_{1}+T_{2}\right)+2 \mu
$$




$$
\beta \times\left(T_{1}^{\prime \prime}-T_{2}^{\prime \prime}\right)=3\left(T_{1}-T_{2}\right)
$$

We introduce new constants $T_{a}=\frac{T_{1}+T_{2}}{2}, T_{C}=\frac{T_{1}-T_{2}}{2}$.

Then we get

$$
\begin{gathered}
\beta \times T_{a}^{\prime \prime}=9 T_{a}+\mu \\
\beta \times T_{c}^{\prime \prime}=3 T_{c} \\
T_{a}^{\prime \prime}=\frac{9}{\beta} T_{a}+\frac{\mu}{\beta}\left(5^{*}\right) \\
T_{c}^{\prime \prime}=\frac{3}{\beta} T_{c}
\end{gathered}
$$

We denote by $\lambda_{1}^{2}=\frac{9}{\beta}, \lambda_{2}^{2}=\frac{3}{\beta}, \gamma_{1}=\frac{1}{\beta}$. Then

$$
\begin{gathered}
T_{a}^{\prime \prime}=\lambda_{1}^{2} \times T_{a}+\gamma_{1} \mu \\
T_{c}^{\prime \prime}=\lambda_{2}^{2} T_{c} .
\end{gathered}
$$

Border conditions $\mathrm{T} 1(0)=\mathrm{T} 1(1)=0, \mathrm{~T} 2(0)=\mathrm{T} 2(1)=0$, or $\mathrm{Ta}(0)=\mathrm{Ta}(1)=\mathrm{Tc}(0)=\mathrm{Tc}(1)=0$, where 1 - half-span.

The solution of system (6) has the form

$T_{a}=A \times \operatorname{sh} \lambda_{1} X+B \times \operatorname{ch} \lambda_{1} X+\frac{\gamma_{1}}{\lambda_{1}} \int_{0}^{x} \mu(\xi) \operatorname{sh} \lambda_{1}(X-\xi) d \xi$

$T_{c}=C \times \operatorname{sh} \lambda_{2} X+D \times \operatorname{ch} \lambda_{2} X$.

From the boundary conditions $\mathrm{Tc}(0)=\mathrm{Tc}(\mathrm{l})=0$ should be $\mathrm{Tc}=0, \mathrm{~T}=\mathrm{Ta}=\mathrm{T} 1=\mathrm{T} 2$.

Total shearing forces in the joint will be:

$$
\begin{array}{ll}
0 \leq \mathrm{X} \leq \mathrm{a} & T_{a}=\frac{h \gamma_{1}}{3 i^{2}} \times \frac{P}{\lambda_{1}^{2}}\left[X-\frac{\operatorname{ch} \lambda_{1}(l-a) \times \operatorname{sh} \lambda_{1} X}{\lambda_{1} \times \operatorname{ch} \lambda_{1} l}\right] \\
\mathrm{a} \leq \mathrm{X} \leq \mathrm{l} & T_{a}=\frac{h \gamma_{1}}{3 i^{2}} \times \frac{P}{\lambda_{1}^{2}}\left[a+\frac{\operatorname{sh} \lambda_{1}(x-a)}{\lambda_{1}^{2}}-\frac{\operatorname{ch} \lambda_{1}(l-a)}{\operatorname{ch} \lambda_{1} l} \times \frac{\operatorname{sh} \lambda_{1} X}{\lambda_{1}}\right] .
\end{array}
$$

Running forces in the joints are calculated by formulas $\tau=T^{\prime}$ :

$$
\begin{aligned}
\tau_{1} & =\frac{h \gamma_{1}}{3 i^{2}} \times \frac{P}{\lambda_{1}^{2}}\left[1-\frac{\operatorname{ch} \lambda_{1}(l-a) \times \operatorname{ch} \lambda_{1} X}{\operatorname{ch} \lambda_{1} l}\right] \text { when } 0 \leq \mathrm{X} \leq \mathrm{a}, \\
\tau_{2} & =\frac{h \gamma_{1}}{3 i^{2}} \times \frac{P}{\lambda_{1}^{2}}\left[\operatorname{ch} \lambda_{1}(x-a)-\frac{\operatorname{ch} \lambda_{1}(l-a)}{\operatorname{ch} \lambda_{1} l} \times \operatorname{ch} \lambda_{1} X\right]
\end{aligned}
$$

when $\mathrm{a} \leq \mathrm{X} \leq 1$, where $\lambda_{1}^{2}=\frac{9}{\beta}, \beta=\frac{E F}{\xi}, \gamma_{1}=\frac{1}{\beta}, i^{2}=\frac{\mathrm{I}}{F}=\frac{h^{2}}{12}$.

We substitute into equations:

$$
\begin{gathered}
\tau_{1}=\frac{4 P}{9 h}\left[1-\frac{\operatorname{ch} \lambda_{1}(l-a) \times \operatorname{ch} \lambda_{1} X}{\operatorname{ch} \lambda_{1} l}\right] \text { when } 0 \leq \mathrm{X} \leq \mathrm{a}, \\
\tau_{2}=\frac{4 P}{9 h}\left[\operatorname{ch} \lambda_{1}(x-a)-\frac{\operatorname{ch} \lambda_{1}(l-a)}{\operatorname{ch} \lambda_{1} l} \times \operatorname{ch} \lambda_{1} X\right] \text { when } \mathrm{a} \leq \mathrm{X} \leq 1 .
\end{gathered}
$$


The displacements (deflections) of the beam are calculated by the formula:

$\sum_{i=1}^{3} E_{i} I_{i} W^{\prime \prime}=-M^{0}+\sum h_{i} \times T_{i}$
$\sum_{i=1}^{3} E_{i} I_{i} W=\iint\left(M^{0}-2 h T_{1}\right) d X^{2}+C_{1} X+C_{2}$.

For the accepted loading scheme on the section $0 \leq \mathrm{X} \leq \mathrm{a}$, where $M^{0}=P \times X$

$\sum_{i=1}^{3} E_{i} I_{i} W_{1}=-\iint[P X$

$$
\begin{aligned}
& \left.-2 h \frac{h \gamma_{1}}{3 i^{2}} \times \frac{P}{\lambda_{1}^{2}} \times\left(X-\frac{\operatorname{ch} \lambda_{1}(l-a) \times \operatorname{sh} \lambda_{1} X}{\lambda_{1} \times \operatorname{ch} \lambda_{1} l}\right)\right] d X^{2}+C_{1} X \\
& +C_{2} .
\end{aligned}
$$

$\sum_{i=1}^{3} E_{i} I_{i} W_{1}^{\prime}=-\frac{P X^{2}}{2}+\frac{8}{9} P \times\left(\frac{X^{2}}{2}-\frac{\operatorname{ch} \lambda_{1}(l-a) \times \operatorname{ch} \lambda_{1} X}{\operatorname{ch} \lambda_{1} l \times \lambda_{1}^{2}}\right)+C_{1}$.

$\sum_{i=1}^{3} E_{i} I_{i} W_{1}=-\frac{P X^{3}}{6}+\frac{8}{9} P \times\left(\frac{X^{3}}{6}-\frac{\operatorname{ch} \lambda_{1}(l-a) \times \operatorname{sh} \lambda_{1} X}{\operatorname{ch} \lambda_{1} l \times \lambda_{1}^{3}}\right)+C_{1} X+C_{2}$.

$$
\begin{aligned}
& \text { When } \mathrm{a} \leq \mathrm{X} \leq 1 \text { и } M^{0}=P \times a \\
& \begin{aligned}
\sum_{i=1}^{3} E_{i} I_{i} W_{2}=-\iint[P a \\
\\
\left.\quad-2 h \frac{h \gamma_{1}}{3 i^{2}} \times \frac{P}{\lambda_{1}^{2}} \times\left(a-\frac{\operatorname{sh} \lambda_{1}(x-a)}{\lambda_{1}}-\frac{\operatorname{ch} \lambda_{1}(l-a) \times \operatorname{sh} \lambda_{1} X}{\operatorname{ch} \lambda_{1} l \times \lambda_{1}}\right)\right] d X^{2} \\
+C_{3} X+C_{4} .
\end{aligned}
\end{aligned}
$$$$
\sum_{i=1}^{3} E_{i} I_{i} W_{2}^{\prime}=-P a X
$$$$
+\frac{8}{9} P \times\left(a X+\frac{\operatorname{ch} \lambda_{1}(X-a)}{\lambda_{1}^{2}}-\frac{\operatorname{ch} \lambda_{1}(l-a) \times \operatorname{ch} \lambda_{1} X}{\operatorname{ch} \lambda_{1} l \times \lambda_{1}^{2}}\right)+C_{3} .
$$$$
\sum_{i=1}^{3} E_{i} I_{i} W_{2}=-\frac{P a X^{2}}{2}+\frac{8}{9} P \times\left(\frac{a X^{2}}{2}+\frac{\operatorname{sh} \lambda_{1}(X-a)}{\lambda_{1}^{2}}-\frac{\operatorname{ch} \lambda_{1}(l-a) \times \operatorname{sh} \lambda_{1} X}{\operatorname{ch} \lambda_{1} l \times \lambda_{1}^{3}}\right)+
$$$$
C_{1} X+C_{2} \text {. }
$$

The arbitrary integration constants $\mathrm{Ci}$ are determined from the boundary conditions:

$$
C_{1}=-P \times\left(\frac{a^{2}}{18}-\frac{8}{9 \lambda_{1}^{2}}+\frac{a l}{9}\right), C_{2}=0, \quad C_{3}=\frac{1}{9} P a l, C_{4}=P a \times\left(\frac{a^{2}}{54}-\frac{8}{9 \lambda_{1}^{2}}\right) .
$$

\section{Results}

On the basis of the expressions obtained, let us consider the effect of humidity on the load-bearing capacity of wooden beams according to the criteria of the second group of 
limiting states. The results of the calculations are presented in Table 1 and in the graphs in Fig. 1 and Fig. 2.

Table 1. Calculated deflections of a wooden beam of a composite section

\begin{tabular}{|c|c|c|c|c|c|c|}
\hline \multirow[t]{2}{*}{ № } & \multirow{2}{*}{$\begin{array}{c}\text { Load on the } \\
\text { structure } 2 * P \text {, } \\
\text { кH }\end{array}$} & \multicolumn{5}{|c|}{$\begin{array}{l}\text { The calculated deflections } f, \mathrm{~mm} \text { in the middle of the } \\
\text { span, at humidity of wood } \mathrm{W} \%\end{array}$} \\
\hline & & 6 & 12 & 18 & 24 & 30 \\
\hline 1 & 20 & 5.81 & 6.34 & 6.97 & 7.80 & 8.93 \\
\hline 2 & 40 & 12.91 & 14.02 & 15.30 & 16.97 & 19.25 \\
\hline 3 & 60 & 20.99 & 22.65 & 24.59 & 27.12 & 30.55 \\
\hline 4 & 80 & 29.96 & 32.19 & 34.81 & 38.22 & 42.82 \\
\hline 5 & 100 & 39.76 & 42.62 & 45.94 & 50.24 & 56.04 \\
\hline \multicolumn{3}{|c|}{ Elastic } & 11522 & 10162 & 8800 & 7440 \\
\hline
\end{tabular}

Moisture of wood 6-8\% corresponds to the dry mode of premises in which the operation of wooden structures is carried out at relative humidity up to $40 \%$. For the reference point, the deflections of the structure are taken with the standard wood moisture $\mathrm{W}=12 \%$. Moisture of wood $18 \%$ corresponds to the operation of structures with a relative air humidity of up to $80 \%$, which is typical for wooden structures in unheated ventilated rooms. Humidity $30 \%$ corresponds to the hygroscopicity limit (fiber saturation point) of wood.

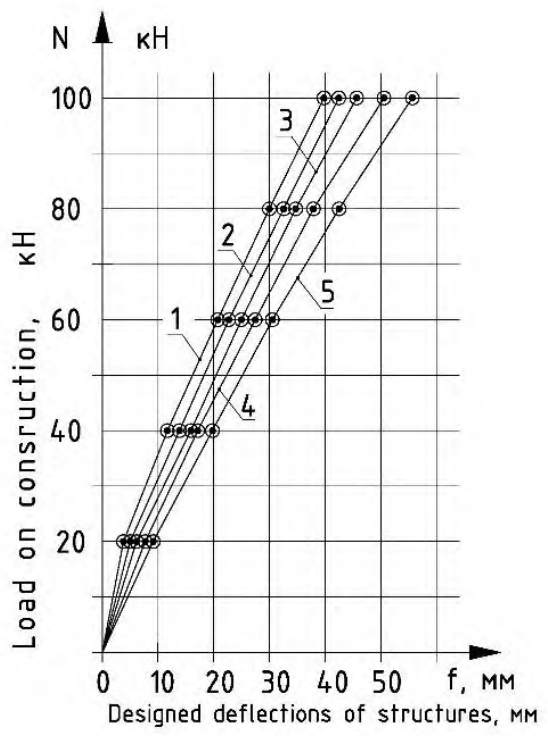

Fig. 1. Estimated deflections of wooden beams of composite cross-section at moisture content of wood: $1-6 \%, 2-12 \%, 3-18 \%, 4-24 \%, 5-30 \%$. 


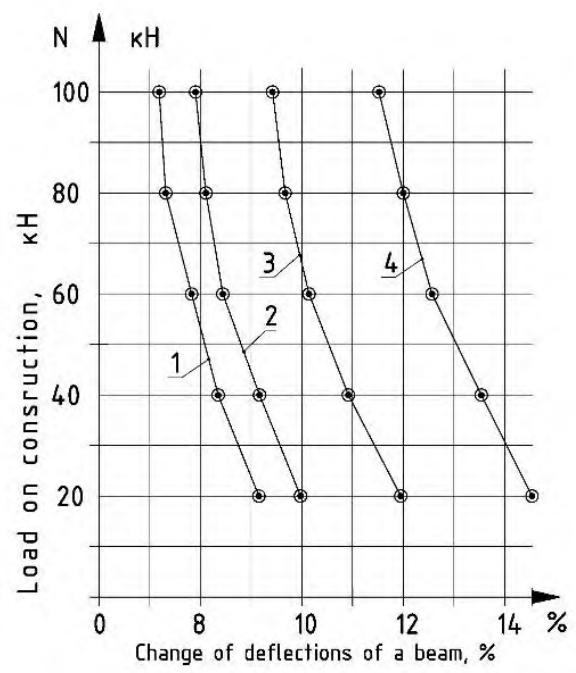

Fig. 2. The change in the calculated deflections of wooden beams of the composite section with increasing wood moisture: $1-6$ to $12 \%, 2-12$ to $18 \%, 3-18$ to $24 \%, 4$ - from 24 to $30 \%$.

\section{Discussion}

From the consideration of Table 1 and the graphs in Fig. 1 and 2, we see that as the moisture content of the wood increases, the deflections of the structure at all loading levels increase. With an increase in wood moisture from $6 \%$ to $12 \%$, the design deflections within the limits of its design load capacity increase by an average of $8.1 \%$. Further, with an increase in humidity from $12 \%$ to $30 \%$ in $6 \%$ increments, the deflections increase by $8.7 \%$, $10.5 \%$, and $12.8 \%$, respectively. At the same time, with increasing load, the intensity of increment of deflections with increasing moisture content of wood decreases, but by no more than $2.6 \%$. Taking the value of the ultimate deflection $\mathrm{fu}=1 / 200 \mathrm{~L}=30 \mathrm{~mm}$, we can establish that the use of structures from wood of natural moisture, which is one of the advantages of glueless compounds, including connections on inclined metal rods, requires reducing the design load-carrying capacity of structures according to the criteria of the second group of limiting states in 1.33 times. The method used in this article is same to referenced works [7-10].

\section{Conclusion}

Based on the calculations performed, the following conclusions are drawn:

1. Using the theory of composite rods AR Rzahynitsyn, the effect of wood moisture on the load-carrying capacity of wooden beams of a composite section on compliant bonds in the second group of limit states.

2. The calculated values of deflections are obtained, taking into account the change in the modulus of elasticity of the structural material when the moisture content of the wood changes. Deflections at the level of design load-bearing capacity of the structure from the 
condition of normal stresses, which were determined at a standard wood moisture content $\mathrm{W}=12 \%$, amounted to $\mathrm{f}=29.96 \mathrm{~mm}=1 / 200 \mathrm{~L}$ with wood moisture $\mathrm{W}=6 \%$ to $\mathrm{f}=42.82$ $\mathrm{mm}$ at a wood moisture content $\mathrm{W}=30 \%$.

3. The use of structures made of natural moisture wood, which is one of the advantages of glueless compounds, to which the connections on inclined metal rods relate, requires a reduction in the design load-carrying capacity of structures according to the criteria of the second group of limiting states 1.33 times.

4. Construction lift attached to the construction of the composite section with connections on inclined metal rods without the use of glue in the manufacturing stage should be at least $1 / 200$ span.

\section{References}

1. V. I. Travush, Journal for Civil and Structural Engineering, 1 (2018)

2. S. Turkovsky, Building materials, 5 (2013)

3. V. Linkov. Building mechanics and calculation of structures, 3 (2017)

4. V. Linkov. Technology of textile industry, 3 (2017)

5. V. Linkov. Construction mechanics and design of structures, 5 (2011)

6. G. Makarov. Science, Education and Experimental Design, 1 (2015)

7. E. Popov, V. Filippov. Forest Journal, 4 (2016)

8. A. Klyukin. Bulletin of the Irkutsk Technical University, 1, (2015)

9. G. Makarov. MARCHI, 1, (2015).

10. V. Zhadanov. Industrial and civil engineering. 11 (2017) 